\title{
EVALUATION OF SELECTED QUALITY CHARACTERISTICS OF FINE GROUND SAUSAGES OF FRANKFURTER TYPE CONTAINING MSPM
}

\author{
OCENA WYBRANYCH WYRÓŻNIKÓW JAKOŚCI KIEŁBAS \\ DROBNO ROZDROBNIONYCH TYPU PARÓWKOWA Z UDZIAŁEM MDOM
}

\begin{abstract}
Summary
Background. More and more poultry is intended for the production of boneless culinary meat both in Poland and other EU countries. The method in which meat is prepared causes inaccurate deboning, leaving a relatively meaningful amount of muscle tissue on the skeletal system. Loss of protein, beneficial for its nutritional value, reaches the level of a few percent of the total of the obtained meat mass. Thus, the issue of mechanically separated poultry meat (MSPM) utilization becomes significant. However, meat mechanically deboned may deteriorate the sensory quality of the final product with respect to characteristics such as taste, odour, consistency, and colour. In this context, the work is focused on an evaluation of selected quality features of the frankfurter type sausage with varied MSPM participation obtained from poultry necks and bodies.

Material and methods. The research material was a frankfurter type sausage, made from class III pork - control sample K. In the other experimental samples, $25 \%$ and $50 \%$ of pork was substituted by MSPM obtained from necks (Sz25 and Sz50) and bodies (W25 and W50) of broilers of the COB breed. In the experimental sausages the following were measured: chemical composition and colour $L * a * b *$ in CIE system. Then, sensory evaluation was carried out applying a 5-point method. The obtained results were subjected to statistical analysis.

Results. On the basis of the obtained results it was stated that the basic composition of the final product changed depending upon the type and amount of MSPM introduced into the raw material composition. The introduction of MSPM into the mentioned meat products composition brought about a significant decrease in the notation of characteristics such as odour, cross-section colour, and exterior colour, measured sensorically. However, the statistical analysis of the results did not show a significant impact of a partial substitution of pork meat with mechanically deboned poultry meat on the overall desirability of the model sausages.

Conclusions. A partial substitution of pork meat with mechanically deboned poultry meat from broilers' necks and bodies is possible. MSPM content of the raw material composition higher than
\end{abstract}


Danyluk, B., Bilska, A., Kowalski, R., Danyluk, M. (2016). Evaluation of selected quality characteristics of fine ground sausages of frankfurter type containing MSPM. Nauka Przyr. Technol., 10, 4, \#42. DOI: http://dx.doi.org/ 10.17306/J.NPT.2016.4.42

$25 \%$ may negatively influence some sensory quality characteristics. No significant impact of substitution of pork meat MSPM on physical colour parameters $a^{*}$ and $b^{*}$ was noticed, independently of the type of MSPM and amount of substitution in the raw material.

Key words: frankfurter type sausage, mechanically separated poultry meat (MSPM), quality

\section{Introduction}

Comminuted homogenized sausages of frankfurter and mortadella type belong to the group of most popular meat products owing to their specific sensory qualities (homogenous structure, consistence, flavour), and an acceptable quality to price ratio of the products on the Polish market. Their production uses less valuable raw materials, having a high fat and connective tissue content, and blood splashing. MSPM, i.e. mechanically separated poultry meat, is frequently used in this type of products (Cegiełka et al., 2014).

MSPM is a raw material obtained in the process of meat separation from the tissues adjacent to the bones by help of mechanical equipment resulting in a total loss or modification of the muscle fibres. MSPM has a smeary consistence, owing to a high degree of residual muscle tissue comminuting, participation of bone marrow and high fat content. The characteristic feature of MSPM is a high calcium and phosphorus content (a few times higher than in the muscle tissue), coming from the periosteum and minute bone chips. MSPM colour is darker than that of muscles. However, a high degree of comminuting, a high content of fat and heme pigments, the presence of metal ions (calcium, iron) and bone marrow may promote unfavourable chemical changes and cause a low microbiological stability of the material. Nevertheless, it needs to be stressed that a raw material of this type is characterized by a relatively high content of protein (9-18\%) (Bełkot et al., 2013). The research carried out so far also indicates that MSPM contains a higher amount of bioavailable fluorine compared to the muscle tissues (Jędra et al., 2001; Stangierski et al., 2011). MSPM can be obtained from chilled or frozen chicken, hen, turkey, duck, and goose carcass elements, as well as complete carcasses passed by the Veterinary Sanitation Department as fit for human consumption (e.g. spent laying hens, carcasses of heavy broiler chicken) (Pomykała and Michalski, 2008; Stangierski et al., 2011).

MSPM production process may proceed with an application of a high-pressure technology, delivering a smeary consistence raw material being of a relatively limited technological use, or low-pressure technology providing a partly preserved structure, yet the technological output of the latter is similar to that of meat scraps manually separated from bones. Taking into consideration the fact that more and more poultry, both in Poland and EU countries, is used for culinary meat production (boneless breast muscles, drumsticks and shanks) utilization of MSPM becomes particularly significant for many producers.

Utilization of the cheap raw material, MSPM, in meat products with a high value added, bears a significant economical value for the EU countries. The aim of the paper was an evaluation of selected characteristics of fine ground sausages of frankfurter type 
Danyluk, B., Bilska, A., Kowalski, R., Danyluk, M. (2016). Evaluation of selected quality characteristics of fine ground sausages of frankfurter type containing MSPM. Nauka Przyr. Technol., 10, 4, \#42. DOI: http://dx.doi.org/ 10.17306/J.NPT.2016.4.42

with a varied participation of MSPM, obtained by high pressure method from necks and carcasses of COB broiler chicken breed.

\section{Material and methods}

Fine ground sausage of frankfurter type was the experimental material. The control sausage batter (control sample K) composition was: pork meat class III (butt) -540 $\mathrm{g} / \mathrm{kg}$, fine fat $-250 \mathrm{~g} / \mathrm{kg}$, water/ice $-210 \mathrm{~g} / \mathrm{kg}$. The "parówki maślane" (butter ground sausage spice) (AmcO) condiment in the quantity $7 \mathrm{~g} / \mathrm{kg}$ of the final product, curing mixture $\left(99.4 \% \mathrm{NaCl} ; 0.6 \% \mathrm{NaNO}_{2}\right)-18 \mathrm{~g} / \mathrm{kg}$, triphosphate pentasodium $-5 \mathrm{~g} / \mathrm{kg}$ (Haifa Chemicals), concentrate of soya proteins - $12 \mathrm{~g} / \mathrm{kg}$ (Arcon ST, ADM). In the other experimental sausage variants, $25 \%$ of pork meat class III was substituted with MSPM from necks (Sz25 and Sz50) and poultry bodies (W25 and W50).

Pork meat and fine fat were pre-comminuted in a grinder (Mainca, type PM-70), applying foreknife, four-armed knife and net with $3 \mathrm{~mm}$ mesh diameter, then it was homogenized in a cutter of Mainca, type CM-21. During cuttering, lasting for about 12 min (rotations $2000 \cdot \mathrm{min}^{-1}$ ), mechanically separated frozen poultry meat was added along with additives and spices. The final temperature of the batter was $14^{\circ} \mathrm{C}$.

Natural casings were filled with the batter (pork chitterlings of $26-28 \mathrm{~mm}$ in diameter) which were turned into $15 \mathrm{~cm}$ long links. The formed links of the experimental meat products were dried for $15 \mathrm{~min}$, at $50^{\circ} \mathrm{C}$, subjected to smoking with $52^{\circ} \mathrm{C}$ hot smoke, next they were warmed with steam up to $72^{\circ} \mathrm{C}$ in the geometric center (steam-smoking chamber Pekmont). The formed sausages were cooled by help of water spray at $16^{\circ} \mathrm{C}$ till $25^{\circ} \mathrm{C}$ interior temperature was reached. Ready products were stored, during the examination, in a chilling room at $4^{\circ} \mathrm{C}$ for $24 \mathrm{~h}$.

The chemical composition of the model sausages was marked according to the Polish Norms: water content - dryer method (acc. PN-ISO 1442:2000, 2000), protein content basing on the marking of amino nitrogen - applying Kjeldahl's method (acc. PN-75/A/04018, 1975), using the automatic destillation set KjelTec (Tecator), fat content - Soxlet method, by help of Soxtec apparatus (Tecator) (acc. PN-ISO 1444:2000, 2000).

An instrumental evaluation was carried out of physical colour parameters L*, a*, b* of the experimental sausages, in CIE system. The research made use of the spectrophotometric apparatus Spectro-Pen, type LMG 161 (Dr Lange, Germany) enabling to measure the components of colour, applying the reflection method within the range of visible light (400-700 nm). Typical for food industry analytics, i.e. standard observer's settings, were applied, i.e. angle of incidence $10^{\circ}$, light $\mathrm{D}_{65}$. Calibration of the appliance, taking into consideration the scattered light and white reference standard LZM 224 Standard 3125 of $\mathrm{X}_{\mathrm{cal}}=92.1, \mathrm{Y}_{\mathrm{cal}}=97.2, \mathrm{Z}_{\mathrm{cal}}=103.9$ parameters, was carried out every time before taking the measurements.

Sensory examination of the experimental sausages, cooled down to $4{ }^{\circ} \mathrm{C}$, was conducted basing on the calibration method, comprising the following range of partial evaluation notes: 5 - very good quality, 4 - good, 3 - satisfactory, 2 - unsatisfactory, 1 bad (Baryłko-Pikielna, ed., 1975). In order to make an evaluation of the overall desirab- 
Danyluk, B., Bilska, A., Kowalski, R., Danyluk, M. (2016). Evaluation of selected quality characteristics of fine ground sausages of frankfurter type containing MSPM. Nauka Przyr. Technol., 10, 4, \#42. DOI: http://dx.doi.org/ 10.17306/J.NPT.2016.4.42

ility of the examined products, the following coefficients of ponderability were used: 0.1 - general appearance, 0.2 - structure and consistence, 0.3 - colour of cross-section, 0.4 - flavour (Bilska et al., 2004).

The obtained results were subjected to statistical analysis. The level of significance of inference $\mathrm{p} \leq 0.05$ was accepted for verifying the research hypotheses. Two-way analysis of variance ANOVA and Fisher multiple comparison test were applied. The evaluations were done using Excel 2010 and Statistica 10.0 computer programs.

\section{Results and discussion}

The carried out investigations proved that the basic composition (water, protein, dry matter and fat content) of fine ground sausages of frankfurter type was not significantly changed, independently of the type and amount of MSPM introduced into the raw material composition. The fat content was not higher than $30 \%$ independently of the type of the accepted technological modification (Table 1). The results gathered in the table clearly indicate that the basic composition was concordant with the non-obligatory PN-A-82007/A1:1998 (1998) norm, which informs that homogenized sausages should contain not less than $9.0 \%$ protein, not more than $40.0 \%$ fat and $69.0 \%$ water.

Table 1. Basic chemical composition of the frankfurter type sausages produced with the participation of MSPM ( $\left.\mathrm{x}_{\text {śr }} \pm \mathrm{s} ; \mathrm{N}=3\right)(\%)$

Tabela 1. Podstawowy skład chemiczny kiełbas typu parówkowa wyprodukowanych z udziałem $\operatorname{MDOM}\left(\mathrm{X}_{\text {śr }} \pm \mathrm{s} ; \mathrm{N}=3\right)(\%)$

\begin{tabular}{|c|c|c|c|}
\hline $\begin{array}{c}\text { Sausage variant } \\
\text { Wariant kiełbasy }\end{array}$ & $\begin{array}{c}\text { Water } \\
\text { Woda }\end{array}$ & $\begin{array}{c}\text { Fat } \\
\text { Tłuszcz }\end{array}$ & $\begin{array}{c}\text { Total protein } \\
\text { Białko ogółem }\end{array}$ \\
\hline K & $58.17^{\mathrm{a}} \pm 2.26$ & $25.87^{\mathrm{a}} \pm 3.08$ & $11.59^{\mathrm{a}} \pm 1.04$ \\
Sz25 & $60.26^{\mathrm{a}} \pm 1.95$ & $24.99^{\mathrm{a}} \pm 1.37$ & $11.70^{\mathrm{a}} \pm 0.29$ \\
Sz50 & $59.04^{\mathrm{a}} \pm 3.24$ & $26.38^{\mathrm{a}} \pm 2.99$ & $10.59^{\mathrm{a}} \pm 1.44$ \\
W25 & $59.36^{\mathrm{a}} \pm 1.97$ & $24.59^{\mathrm{a}} \pm 2.22$ & $11.70^{\mathrm{a}} \pm 0.54$ \\
W50 & $59.35^{\mathrm{a}} \pm 1.95$ & $28.11^{\mathrm{a}} \pm 7.18$ & $11.69^{\mathrm{a}} \pm 1.19$ \\
\hline
\end{tabular}

$\mathrm{X}_{\text {śr }}-$ mean value, $\mathrm{s}-$ standard deviation.

Different letters in the same column indicate statistically significant differences $(p \leq 0.05)$.

$\mathrm{X}_{\text {śr }}$ - wartość średnia, $\mathrm{s}$ - odchylenie standardowe.

Różne litery w tej samej kolumnie wskazują na różnice istotne statystycznie $(\mathrm{p} \leq 0,05)$.

The used raw materials composition did not significantly differentiate the experimental sausages as far as the colour parameters $a^{*}$ and $b^{*}$ were concerned. An exception was parameter $\mathrm{a}^{*}$ of the Sz50 variant on the $6^{\text {th }}$ day of storing whose value was significantly lower than in the case of W50 variant (Table 2). Significant differences were found, though, between the mean values of sausage colour lightness after $24 \mathrm{~h}$ of chilling room storing. $\mathrm{L}^{*}$ value of the control sample colour was significantly different 
Danyluk, B., Bilska, A., Kowalski, R., Danyluk, M. (2016). Evaluation of selected quality characteristics of fine ground sausages of frankfurter type containing MSPM. Nauka Przyr. Technol., 10, 4, \#42. DOI: http://dx.doi.org/ 10.17306/J.NPT.2016.4.42

Table 2. Colour parameters of the frankfurter type sausages produced with the participation of MSPM stored in chilling conditions $\left(\mathrm{x}_{\text {śr }} \pm \mathrm{s} ; \mathrm{N}=3\right)$

Tabela 2. Parametry barwy kiełbas typu parówkowa wyprodukowanych z udziałem MDOM przechowywanych $\mathrm{w}$ warunkach chłodniczych $\left(\mathrm{x}_{\text {śr }} \pm \mathrm{s} ; \mathrm{N}=3\right)$

\begin{tabular}{|c|c|c|c|c|}
\hline $\begin{array}{c}\text { Storage time } \\
\text { (days) } \\
\text { Czas przechowywania } \\
\text { (dni) }\end{array}$ & $\begin{array}{l}\text { Sausage variant } \\
\text { Wariant kiełbasy }\end{array}$ & $\mathrm{L}^{*}$ & $a^{*}$ & $\mathrm{~b}^{*}$ \\
\hline \multirow[t]{5}{*}{1} & $\mathrm{~K}$ & $69.43^{\mathrm{c}} \pm 2.89$ & $7.00^{\mathrm{ab}} \pm 0.30$ & $12.73^{\mathrm{abc}} \pm 1.55$ \\
\hline & Sz25 & $61.25^{\mathrm{a}} \pm 13.97$ & $7.62^{\mathrm{ab}} \pm 1.81$ & $14.07^{\mathrm{bc}} \pm 1.40$ \\
\hline & Sz50 & $66.31^{\mathrm{abc}} \pm 6.30$ & $6.19^{\mathrm{ab}} \pm 1.38$ & $14.07^{\mathrm{bc}} \pm 0.86$ \\
\hline & W25 & $66.07^{\mathrm{abc}} \pm 3.71$ & $8.31^{\mathrm{b}} \pm 0.97$ & $1.83^{\mathrm{abc}} \pm 1.16$ \\
\hline & W50 & $61.98^{\mathrm{ab}} \pm 7.85$ & $8.33^{b} \pm 1.81$ & $14.41^{\mathrm{c}} \pm 0.48$ \\
\hline \multirow[t]{5}{*}{4} & K & $69.03^{\mathrm{bc}} \pm 2.32$ & $7.28^{\mathrm{ab}} \pm 0.44$ & $12.32^{\mathrm{ab}} \pm 0.43$ \\
\hline & Sz25 & $6.26^{\mathrm{c}} \pm 1.80$ & $6.26^{\mathrm{ab}} \pm 1.41$ & $12.73^{\mathrm{abc}} \pm 1.31$ \\
\hline & Sz50 & $68.20^{\mathrm{abc}} \pm 3.63$ & $6.20^{\mathrm{ab}} \pm 1.60$ & $14.25^{\mathrm{bc}} \pm 1.55$ \\
\hline & W25 & $67.21^{\mathrm{abc}} \pm 2.85$ & $7.89^{\mathrm{ab}} \pm 0.78$ & $12.52^{\mathrm{abc}} \pm 0.88$ \\
\hline & W50 & $66.10^{\mathrm{abc}} \pm 2.07$ & $8.00^{\mathrm{ab}} \pm 1.73$ & $13.80^{\mathrm{abc}} \pm 1.13$ \\
\hline \multirow[t]{5}{*}{6} & K & $70.32^{\mathrm{c}} \pm 1.52$ & $7.08^{\mathrm{ab}} \pm 0.49$ & $11.90^{\mathrm{a}} \pm 1.19$ \\
\hline & Sz25 & $68.47^{\mathrm{bc}} \pm 1.93$ & $6.53^{\mathrm{ab}} \pm 1.26$ & $12.84^{\mathrm{abc}} \pm 1.30$ \\
\hline & Sz50 & $69.56^{\mathrm{c}} \pm 2.53$ & $5.85^{\mathrm{a}} \pm 1.04$ & $13.52^{\mathrm{abc}} \pm 2.53$ \\
\hline & W25 & $67.96^{\mathrm{abc}} \pm 0.89$ & $7.90^{\mathrm{ab}} \pm 0.52$ & $12.74^{\mathrm{abc}} \pm 1.14$ \\
\hline & W50 & $66.27^{\mathrm{abc}} \pm 1.36$ & $8.14^{b} \pm 1.45$ & $13.70^{\mathrm{abc}} \pm 0.72$ \\
\hline \multirow[t]{5}{*}{8} & K & $69.24^{\mathrm{c}} \pm 0.74$ & $6.18^{\mathrm{ab}} \pm 2.43$ & $12.25^{\mathrm{ab}} \pm 0.81$ \\
\hline & Sz25 & $69.99^{\mathrm{c}} \pm 0.80$ & $6.93^{\mathrm{ab}} \pm 0.96$ & $12.84^{\mathrm{abc}} \pm 0.51$ \\
\hline & Sz50 & $69.25^{\mathrm{c}} \pm 2.53$ & $6.15^{\mathrm{ab}} \pm 1.01$ & $13.51^{\mathrm{abc}} \pm 1.86$ \\
\hline & W25 & $68.79^{\mathrm{bc}} \pm 0.88$ & $7.62^{\mathrm{ab}} \pm 1.19$ & $12.83^{\mathrm{abc}} \pm 0.91$ \\
\hline & W50 & $66.73^{\mathrm{abc}} \pm 0.95$ & $7.83^{\mathrm{ab}} \pm 1.80$ & $13.77^{\mathrm{abc}} \pm 1.35$ \\
\hline
\end{tabular}

Explanatory notes - as in Table 1 .

Objaśnienia - jak pod tabelą 1 .

from the value of the sample Sz25 colour lightness $\left(\mathrm{L}^{*}=61.25\right)$ and W50 ( $\left.\mathrm{L}^{*}=61.98\right)$. However, the differences turned out to be insignificant in successive storing periods.

Tolik et al. (2015) in their work, similarly to the below work, found no effect of the type of the applied raw meat (MSPM/MSPM with partly preserved structure) on colour 
6

Danyluk, B., Bilska, A., Kowalski, R., Danyluk, M. (2016). Evaluation of selected quality characteristics of fine ground sausages of frankfurter type containing MSPM. Nauka Przyr. Technol., 10, 4, \#42. DOI: http://dx.doi.org/ 10.17306/J.NPT.2016.4.42

parameters of sterilized pates. As has been proved by numerous investigations so far, various content of heme pigments in meat-fat raw material will not always evoke significant changes in the colour of the final product, owing to its considerable comminuting and mixing of batter components (Trindade et al., 2005).

The results of the sensory examination indicate a significant deterioration of colour quality, both exterior and the cross-section colour, as well as the odour of the sausages in which pork was substituted with MSPM (Table 3). 8 days after the production storing of the experimental sausages: Sz50 and W50 scored an average note of 4.07 points, but the colour of the control variant scored significantly higher points -4.53 points. On the

Table 3. Sensory analysis of the frankfurter type sausages produced with the participation of MSPM stored in chilling conditions ( $\mathrm{X}$ śr $\pm \mathrm{s} ; \mathrm{N}=3$ )

Tabela 3. Analiza sensoryczna kiełbas typu parówkowa wyprodukowanych z udziałem MDOM przechowywanych $\mathrm{w}$ warunkach chłodniczych ( $\mathrm{x}$ śr $\pm \mathrm{s} ; \mathrm{N}=3)$

\begin{tabular}{|c|c|c|c|c|c|c|c|c|}
\hline $\begin{array}{c}\text { Sausage } \\
\text { variant } \\
\text { Wariant } \\
\text { kiełbasy }\end{array}$ & $\begin{array}{c}\text { Outward } \\
\text { appearance } \\
\text { Wygląd } \\
\text { zewnętrzny }\end{array}$ & $\begin{array}{c}\text { Colour } \\
\text { outside } \\
\text { Barwa } \\
\text { zewnętrzna }\end{array}$ & $\begin{array}{l}\text { Colour for } \\
\text { cross- } \\
\text {-section } \\
\text { Barwa na } \\
\text { przekroju }\end{array}$ & $\begin{array}{l}\text { Odour } \\
\text { Zapach }\end{array}$ & $\begin{array}{l}\text { Taste } \\
\text { Smak }\end{array}$ & $\begin{array}{l}\text { Consist- } \\
\text { ency } \\
\text { Konsysten- } \\
\text { cja }\end{array}$ & $\begin{array}{l}\text { Juiciness } \\
\text { Soczystość }\end{array}$ & $\begin{array}{l}\text { Overall } \\
\text { desirability } \\
\text { Ogólna } \\
\text { pożądal- } \\
\text { ność }\end{array}$ \\
\hline 1 & 2 & 3 & 4 & 5 & 6 & 7 & 8 & 9 \\
\hline \multicolumn{9}{|c|}{1 day - 1 dzień } \\
\hline K & 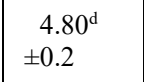 & $\begin{aligned} & 4.80^{\text {cde }} \\
\pm & 0.2\end{aligned}$ & $\begin{aligned} & 4.70^{\mathrm{Bc}} \\
\pm & 0.2\end{aligned}$ & $\begin{aligned} & 4.80^{\mathrm{de}} \\
\pm & 0.2\end{aligned}$ & $\begin{aligned} & 4.57^{\mathrm{c}} \\
\pm & 0.5\end{aligned}$ & $\begin{aligned} & 4.40^{\mathrm{ab}} \\
\pm & 0.6\end{aligned}$ & $\begin{aligned} & 4.47^{\mathrm{ab}} \\
\pm & 0.4\end{aligned}$ & 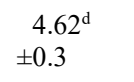 \\
\hline Sz25 & $\begin{aligned} & 4.67^{\mathrm{cd}} \\
\pm & 0.3\end{aligned}$ & $\begin{aligned} & 4.80^{\text {cde }} \\
\pm & 0.2\end{aligned}$ & $\begin{aligned} & 4.33^{\mathrm{Abc}} \\
\pm & 0.2\end{aligned}$ & $\begin{aligned} & 4.73^{\text {cde }} \\
\pm & 0.1\end{aligned}$ & $\begin{aligned} & 4.03^{\mathrm{abc}} \\
\pm & 0.7\end{aligned}$ & $\begin{aligned} & 4.27^{\mathrm{ab}} \\
\pm & 0.9\end{aligned}$ & $\begin{aligned} & 4.47^{\mathrm{ab}} \\
\pm & 0.5\end{aligned}$ & 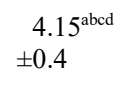 \\
\hline Sz50 & $\begin{array}{l}4.67^{\mathrm{cd}} \\
\pm 0.2\end{array}$ & $\begin{aligned} & 4.7^{\text {bcde }} \\
\pm & 0.4\end{aligned}$ & $\begin{aligned} & 4.33^{\mathrm{Abc}} \\
\pm & 0.2\end{aligned}$ & 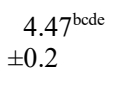 & $\begin{array}{l}3.53^{\mathrm{abc}} \\
\pm 0.8\end{array}$ & $\begin{aligned} & 3.57^{\mathrm{ab}} \\
\pm & 1.3\end{aligned}$ & $\begin{aligned} & 4.17^{\mathrm{ab}} \\
\pm & 0.5\end{aligned}$ & 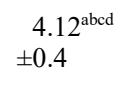 \\
\hline W25 & $\begin{aligned} & 4.60^{\mathrm{bcd}} \\
\pm & 0.0\end{aligned}$ & $\begin{aligned} & 4.83^{\mathrm{de}} \\
\pm & 0.2\end{aligned}$ & $\begin{aligned} & 4.47^{\mathrm{Abc}} \\
\pm & 0.4\end{aligned}$ & $\begin{aligned} & 4.8^{\mathrm{de}} \\
\pm & 0.1\end{aligned}$ & $\begin{aligned} & 3.87^{\mathrm{abc}} \\
\pm & 0.4\end{aligned}$ & $\begin{array}{l}4.07^{\mathrm{ab}} \\
\pm 1.0\end{array}$ & 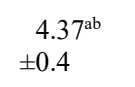 & $\begin{aligned} & 4.37^{\mathrm{abcd}} \\
\pm & 0.3\end{aligned}$ \\
\hline W50 & $\begin{aligned} & 4.77^{\mathrm{cd}} \\
\pm & 0.2\end{aligned}$ & $\begin{aligned} & 4.87^{\mathrm{e}} \\
\pm & 0.1\end{aligned}$ & $\begin{aligned} & 4.23^{\mathrm{Abc}} \\
\pm & 0.3\end{aligned}$ & $\begin{aligned} & 4.27^{\text {abcde }} \\
\pm & 0.7\end{aligned}$ & $\begin{aligned} & 3.27^{\mathrm{a}} \\
\pm & 1.0\end{aligned}$ & $\begin{aligned} & 3.73^{\mathrm{ab}} \\
\pm & 1.4\end{aligned}$ & $\begin{aligned} & 4.03^{\mathrm{ab}} \\
\pm & 0.8\end{aligned}$ & $\begin{aligned} & 4.02^{\mathrm{abcd}} \\
\pm & 0.5\end{aligned}$ \\
\hline \multicolumn{9}{|c|}{4 days -4 dni } \\
\hline $\mathrm{K}$ & $\begin{array}{l}4.73^{\mathrm{cd}} \\
\pm 0.2\end{array}$ & $\begin{array}{l}4.70^{\text {bcde }} \\
\pm 0.3\end{array}$ & 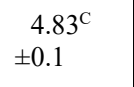 & 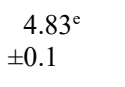 & $\begin{array}{l}4.23^{\mathrm{abc}} \\
\pm 0.9\end{array}$ & $\begin{aligned} & 3.97^{\mathrm{ab}} \\
\pm & 1.0\end{aligned}$ & 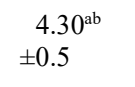 & $\begin{aligned} & 4.49^{\text {bcd }} \\
\pm & 0.4\end{aligned}$ \\
\hline $\mathrm{Sz} 25$ & 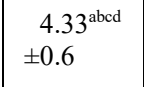 & $\begin{array}{l}4.67^{\text {bcde }} \\
\pm 0.2\end{array}$ & 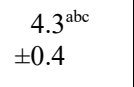 & 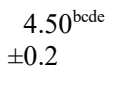 & $\begin{aligned} & 4.07^{\mathrm{abc}} \\
\pm & 0.2\end{aligned}$ & 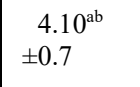 & 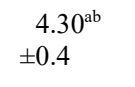 & 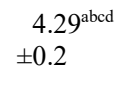 \\
\hline Sz50 & $\begin{aligned} & 4.00^{\mathrm{abc}} \\
\pm & 0.8\end{aligned}$ & 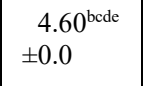 & $\begin{array}{l}4.07^{\mathrm{ab}} \\
\pm 0.6\end{array}$ & 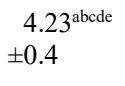 & $\begin{aligned} & 3.50^{\mathrm{ab}} \\
\pm & 0.6\end{aligned}$ & $\begin{aligned} & 3.27^{\mathrm{a}} \\
\pm & 1.2\end{aligned}$ & $\begin{aligned} & 3.97^{\mathrm{a}} \\
\pm & 0.4\end{aligned}$ & $\begin{array}{l}3.90^{\mathrm{abc}} \\
\pm 0.4\end{array}$ \\
\hline W25 & $\begin{array}{l}3.78^{\text {abcd }} \\
\pm 0.6\end{array}$ & $\begin{array}{l}3.90^{\text {bcde }} \\
\pm 0.2\end{array}$ & $\begin{array}{l}4.43^{\mathrm{abc}} \\
\pm 0.4\end{array}$ & $\begin{array}{l}3.77^{\text {bcde }} \\
\pm 0.2\end{array}$ & $\begin{array}{l}3.30^{\mathrm{abc}} \\
\pm 0.3\end{array}$ & $\begin{array}{l}3.40^{\mathrm{ab}} \\
\pm 0.5\end{array}$ & $\begin{array}{l}3.56^{\mathrm{ab}} \\
\pm 0.3\end{array}$ & $\begin{array}{l}4.31^{\mathrm{abcd}} \\
\pm 0.2\end{array}$ \\
\hline W50 & $\begin{aligned} & 4.27^{\mathrm{abcd}} \\
\pm & 0.6\end{aligned}$ & $\begin{aligned} & 4.50^{\text {bcde }} \\
\pm & 0.3\end{aligned}$ & $\begin{array}{l}3.83^{\mathrm{a}} \\
\pm 0.3\end{array}$ & $\begin{aligned} & 3.90^{\mathrm{ab}} \\
\pm & 0.5\end{aligned}$ & $\begin{aligned} & 3.23^{\mathrm{a}} \\
\pm & 0.4\end{aligned}$ & $\begin{aligned} & 3.70^{\mathrm{ab}} \\
\pm & 0.7\end{aligned}$ & 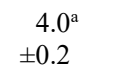 & $\begin{aligned} & 3.80^{\mathrm{ab}} \\
\pm & 0.3\end{aligned}$ \\
\hline
\end{tabular}


Danyluk, B., Bilska, A., Kowalski, R., Danyluk, M. (2016). Evaluation of selected quality characteristics of fine ground sausages of frankfurter type containing MSPM. Nauka Przyr. Technol., 10, 4, \#42. DOI: http://dx.doi.org/ 10.17306/J.NPT.2016.4.42

Table 3 - cont. / Tabela 3 - cd.

\begin{tabular}{|c|c|c|c|c|c|c|c|c|}
\hline 1 & 2 & 3 & 4 & 5 & 6 & 7 & 8 & 9 \\
\hline \multicolumn{9}{|c|}{6 days -6 dni } \\
\hline K & $\begin{array}{l}4.60^{\mathrm{bcd}} \\
\pm 0.4\end{array}$ & $\begin{array}{l}4.77^{\text {bcde }} \\
\pm 0.2\end{array}$ & $\begin{aligned} & 4.67^{\mathrm{bc}} \\
\pm & 0.2\end{aligned}$ & $\begin{aligned} & 4.7^{\text {de }} \\
\pm & 0.1\end{aligned}$ & $\begin{aligned} & 4.43^{\mathrm{bc}} \\
\pm & 0.4\end{aligned}$ & $\begin{aligned} & 4.70^{\mathrm{b}} \\
\pm & 0.1\end{aligned}$ & $\begin{aligned} & 4.50^{\mathrm{ab}} \\
\pm & 0.3\end{aligned}$ & $\begin{aligned} & 4.60^{\text {cd }} \\
\pm & 0.2\end{aligned}$ \\
\hline Sz25 & $\begin{array}{l}4.27^{\mathrm{abcd}} \\
\pm 0.5\end{array}$ & $\begin{array}{l}4.43^{\text {abcd }} \\
\pm 0.2\end{array}$ & 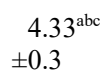 & $\begin{array}{l}4.37^{\text {bcde }} \\
\pm 0.3\end{array}$ & $\begin{array}{l}3.83^{\mathrm{abc}} \\
\pm 0.7\end{array}$ & $\begin{aligned} & 4.37^{\mathrm{ab}} \\
\pm & 0.3\end{aligned}$ & 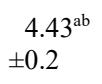 & 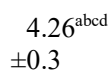 \\
\hline Sz50 & $\begin{array}{l}4.33^{\text {abcd }} \\
\pm 0.4\end{array}$ & $\begin{aligned} & 4.37^{\mathrm{ab}} \\
\pm & 0.3\end{aligned}$ & 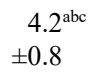 & $\begin{aligned} & 3.97^{\mathrm{ab}} \\
\pm & 0.6\end{aligned}$ & $\begin{array}{l}3.60^{\mathrm{abc}} \\
\pm 0.8\end{array}$ & $\begin{aligned} & 4.0^{\mathrm{ab}} \\
\pm & 0.7\end{aligned}$ & 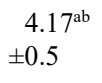 & 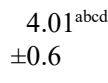 \\
\hline W25 & 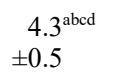 & $\begin{array}{l}4.40^{\mathrm{abc}} \\
\pm 0.2\end{array}$ & 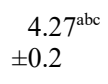 & $\begin{array}{l}4.2^{\mathrm{abcd}} \\
\pm 0.3\end{array}$ & $\begin{array}{l}3.80^{\mathrm{abc}} \\
\pm 0.4\end{array}$ & $\begin{aligned} & 4.10^{\mathrm{ab}} \\
\pm & 0.3\end{aligned}$ & 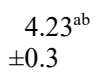 & 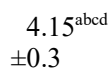 \\
\hline W50 & $\begin{array}{l}4.37^{\text {abcd }} \\
\pm 0.1\end{array}$ & $\begin{array}{l}4.43^{\mathrm{abcd}} \\
\pm 0.1\end{array}$ & $\begin{aligned} & 4.17^{\mathrm{abc}} \\
\pm & 0.4\end{aligned}$ & $\begin{array}{c}3.7^{\mathrm{a}} \\
\pm 0.6\end{array}$ & $\begin{array}{l}3.57^{\mathrm{abc}} \\
\pm 0.5\end{array}$ & $\begin{array}{l}4.0^{\mathrm{ab}} \\
\pm 0.4\end{array}$ & 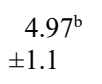 & $\begin{array}{l}3.93^{\text {abcd }} \\
\pm 0.4\end{array}$ \\
\hline \multicolumn{9}{|c|}{8 days -8 dni } \\
\hline K & 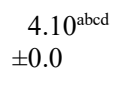 & $\begin{array}{l}4.53^{\text {bde }} \\
\pm 0.1\end{array}$ & 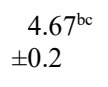 & $\begin{array}{l}4.5^{\text {cde }} \\
\pm 0.1\end{array}$ & $\begin{array}{l}4.20^{\mathrm{abc}} \\
\pm 0.2\end{array}$ & $\begin{aligned} & 4.07^{\mathrm{ab}} \\
\pm & 0.2\end{aligned}$ & 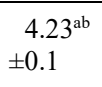 & $\begin{aligned} & 4.34^{\mathrm{abcd}} \\
\pm & 0.1\end{aligned}$ \\
\hline Sz25 & $\begin{array}{l}3.87^{\mathrm{ab}} \\
\pm 0.1\end{array}$ & $\begin{aligned} & 4.40^{\mathrm{bc}} \\
\pm & 0.1\end{aligned}$ & 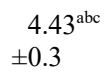 & $\begin{array}{l}4.13^{\mathrm{abc}} \\
\pm 0.3\end{array}$ & $\begin{array}{l}3.73^{\mathrm{abc}} \\
\pm 0.4\end{array}$ & $\begin{aligned} & 3.87^{\mathrm{ab}} \\
\pm & 0.5\end{aligned}$ & $\begin{array}{r}4.0^{\mathrm{a}} \\
\pm 0.4\end{array}$ & $\begin{aligned} & 4.04^{\mathrm{abcd}} \\
\pm & 0.2\end{aligned}$ \\
\hline Sz50 & $\begin{array}{l}3.77^{\mathrm{a}} \\
\pm 0.9\end{array}$ & $\begin{array}{l} \\
\quad 4.07^{\mathrm{a}} \\
\pm 0.5\end{array}$ & $\begin{array}{l}3.87^{\mathrm{a}} \\
\pm 1.0\end{array}$ & $\begin{array}{l}3.9^{\mathrm{ab}} \\
\pm 0.5\end{array}$ & $\begin{array}{l}3.33^{\mathrm{a}} \\
\pm 0.9\end{array}$ & $\begin{array}{r}3.6^{\mathrm{ab}} \\
\pm 1.14\end{array}$ & $\begin{array}{l}3.73^{\mathrm{a}} \\
\pm 0.9\end{array}$ & $\begin{array}{l}3.71^{\mathrm{a}} \\
\pm 0.4\end{array}$ \\
\hline W25 & $\begin{array}{l}4.17^{\mathrm{abcd}} \\
\pm 0.2\end{array}$ & $\begin{array}{l}\text { 4. } 47^{7 \text { abcde }} \\
\pm 0.2\end{array}$ & $\begin{aligned} & 4.23^{\mathrm{abc}} \\
\pm & 0.8\end{aligned}$ & $\begin{array}{l}4.3^{\text {abcde }} \\
\pm 0.2\end{array}$ & $\begin{array}{l}3.6^{\text {abc }} \\
\pm 0.4\end{array}$ & $\begin{array}{r}3.8^{\mathrm{ab}} \\
\pm 0.62\end{array}$ & $\begin{array}{l}3.87^{\mathrm{a}} \\
\pm 0.4\end{array}$ & $\begin{aligned} & 4.01^{\mathrm{abcd}} \\
\pm & 0.4\end{aligned}$ \\
\hline W50 & $\begin{array}{l}4.03^{\text {abcd }} \\
\pm 0.8\end{array}$ & 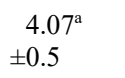 & $\begin{aligned} & 4.20^{\mathrm{abc}} \\
\pm & 0.6\end{aligned}$ & 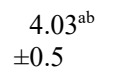 & $\begin{array}{l}3.33^{\mathrm{a}} \\
\pm 0.8\end{array}$ & $\begin{array}{r}3.5^{\mathrm{ab}} \\
\pm 1.22\end{array}$ & $\begin{array}{l}3.67^{\mathrm{a}} \\
\pm 0.9\end{array}$ & $\begin{array}{l}3.78^{\mathrm{ab}} \\
\pm 0.7\end{array}$ \\
\hline
\end{tabular}

Explanatory notes - as in Table 1 .

Objaśnienia - jak pod tabelą 1 .

$4^{\text {th }}$ day of the examination, in case of the variants: Sz50 and W50, a significant deterioration of cross-section colour desirability was observed compared to the control sausage after 4-day storing.

Similar to the assessment of the colour desirability, addition of MSPM into the raw material composition of experimental products influenced the observed decrease in the sensory desirability of odour, in respect to the products, compared to the control sausage variant. The odour of variant W50 sausage was assessed as significantly less desired after 4- and 6-day cool storing, compared to the control sample which mean scores were 4.83 points after 4 days and 4.7 points after 6 days. Despite the reported differences between the mean score values of sensory taste desirability of the examined products, a significant differentiation of the experimental sausages was observed only after $24 \mathrm{~h}$ of post-production storing in the case of variant W50 and the control one. Continued storing (for 4, 6, 8 days) did not meaningfully establish the difference.

The analysis of the obtained results made it possible to state that both the type of the mechanically separated poultry meat and the proportion of the pork meat substituted 
Danyluk, B., Bilska, A., Kowalski, R., Danyluk, M. (2016). Evaluation of selected quality characteristics of fine ground sausages of frankfurter type containing MSPM. Nauka Przyr. Technol., 10, 4, \#42. DOI: http://dx.doi.org/ 10.17306/J.NPT.2016.4.42

with MSPM did not differentiate the sensory desirability of the consistence and juiciness of the examined products.

On the first day after the completion of the production process, statistically significant lower point scores were noted for taste for variant W50 (3.27 pts.), compared to the control variant (4.57). During a longer storing period (4, 6, 8 days) no such differences were reported. Moreover, it was noticed that independently of the experimental sausages variant, storing time had no statistically significant impact on the overall evaluation. The investigations of other authors indicate that using MSPM for homogenized sausages production does not decrease the sensory desirability of the final product (Abdullah, 2007; Cegiełka et al., 2014).

\section{Conclusions}

1. Mechanically separated poultry meat may be a substitution for pork meat in the production technology of cuttered fine ground sausages of frankfurter type.

2. Substitution of pork meat with MSPM, independently of its origin, in the content not higher than $25 \%$ of the recipe mass, did not significantly influence deterioration of the characteristics of the sensory quality of the examined sausages.

3. Long-period storing (longer than 6 days), especially when $50 \%$ of pork meat was substituted, significantly influenced the lower score points in the aspect of sensorically evaluated characteristics such as colour and vapour.

4. Independently of the type and level of pork meat substitution with MSPM, no significant influence of the substitution on the overall desirability of the examined sausages was noticed.

\section{References}

Abdullah, B. M. (2007). Properties of five canned luncheon meat formulations as affected by quality of raw materials. Int. J. Food Sci. Technol., 42, 1, 30-35. DOI: 10.1111/j.1365-2621. 2006.01195.x

Baryłko-Pikielna, N. (ed.). (1975). Zarys analizy sensorycznej żywności. Warszawa: WNT.

Bełkot, Z., Ziomek, M., Gondek, M. (2013). Wartość odżywcza odzyskanego mechanicznie mięsa kurcząt i gęsi. Med. Wet., 69, 8, 499-504.

Bilska, A., Krysztofiak, K., Uchman, W., Woroch, K. (2004). Wpływ rodzaju osłonki i metody pakowania na jakość sensoryczną kiełbasy typu parówkowa. Acta Sci. Pol. Technol. Aliment., $3,1,145-155$.

Cegiełka, A., Kuczyńska, N., Pietrzak, D. (2014). Zastąpienie surowca wieprzowo-wołowego w kiełbasach homogenizowanych przez mięso drobiowe oddzielone mechanicznie, uzyskane po separacji wysoko- i niskociśnieniowej. Żywn. Nauka Technol. Jakość, 94, 3, 123-135. DOI: $10.15193 /$ zntj/2014/94/123-135

Florowski, T., Adamczak, L., Hernández, I. F., Franco, M. B. M., Tyburcy, A. (2010). Ocena wpływu stopnia substytucji tłuszczu inuliną na wybrane wyróżniki jakości modelowych kiełbas. Nauka Przyr. Technol., 4, 5, \#57. 
Danyluk, B., Bilska, A., Kowalski, R., Danyluk, M. (2016). Evaluation of selected quality characteristics of fine ground sausages of frankfurter type containing MSPM. Nauka Przyr. Technol., 10, 4, \#42. DOI: http://dx.doi.org/ 10.17306/J.NPT.2016.4.42

Jędra, M., Urbanek-Karłowska, B., Fonberg-Broczek, M., Sawilska-Rautenstrauch, D., Badowski, P. (2001). Bioavailable fluoride in poultry deboned meat and meat products. Rocz. PZH, 3, 225230.

Makała, H., Olkiewicz, M., Moch, P. (2006). Characteristics of sensory quality of Bologna-types sausage from Polish and Italian markets. Acta Sci. Pol. Technol. Aliment., 5, 2, 107-116.

PN-75/A-04018. (1975). Produkty rolniczo-żywnościowe - Oznaczanie azotu metodą Kjeldahla i przeliczanie na białko. Warszawa: PKNiM.

PN-A-82007/A1:1998. (1998). Przetwory mięsne - Wędliny (Zmiana A1). Warszawa: PKN.

PN-ISO 1442:2000. (2000). Mięso i przetwory mięsne. Oznaczanie zawartości wody. Warszawa: PKN.

PN-ISO 1444:2000. (2000). Mięso i przetwory mięsne. Oznaczanie zawartości tłuszczu wolnego. Warszawa: PKN.

Pomykała, R., Michalski, M. (2008). Jakość mikrobiologiczna mięsa drobiowego oddzielonego mechanicznie. Acta Sci. Pol. Med. Vet., 7, 4, 43-49.

Stangierski, J., Kijowski, J., Konieczny, P. (2011). Jakość i wykorzystanie mięsa drobiowego oddzielonego mechanicznie. Zesz. Nauk. UE Pozn., 205, 202-210.

Tolik, D., Słowiński, M., Desperak, K. (2015). Wpływ zastosowania drobiowego mięsa oddzielonego mechanicznie oraz mięsa odścięgnionego na jakość pasztetów sterylizowanych. Żywn. Nauka Technol. Jakość, 102, 5, 132-141. DOI: 10.15193/zntj/2015/102/077

Trindade, M. A., Contreras, C. C., de Felício, P. E. (2005). Mortadella sausage formulations with partial and total replacement of beef and pork backfat with mechanically separated meat from spent layer hens. J. Food Sci., 70, 3, S236-S241.

Tyburcy, A., Toszek, E., Cegiełka, A. (2005). Porównanie składu surowcowego i wskaźników chemicznych parówek drobiowych i wieprzowych oferowanych w sprzedaży detalicznej w Warszawie. Żywn. Nauka Technol. Jakość, 44, 3, 105-112.

\section{OCENA WYBRANYCH WYRÓŻNIKÓW JAKOŚCI KIEŁBAS DROBNO ROZDROBNIONYCH TYPU PARÓWKOWA Z UDZIAŁEM MDOM}

\footnotetext{
Streszczenie

Wstęp. W Polsce i innych krajach Unii Europejskiej coraz więcej drobiu przeznacza się do produkcji kulinarnego mięsa pozbawionego kości. Sposób jego pozyskiwania powoduje, że stosunkowo duża część tkanki mięśniowej pozostaje nieoddzielona od układu szkieletowego. Straty cennego, z punktu widzenia żywieniowego, białka sięgają nawet kilku procent masy pozyskiwanego mięsa, dlatego zagospodarowanie mięsa drobiowego odkostnionego mechanicznie (MDOM) staje się szczególnie istotne. Zastosowanie mięsa oddzielonego mechanicznie może jednak wpłynąć na pogorszenie jakości sensorycznej gotowego wyrobu, m.in. takich wyróżników, jak: smak, zapach, konsystencja, barwa. Celem pracy była ocena wybranych wyróżników jakości kiełbas typu parówkowa ze zróżnicowanym udziałem MDOM pozyskanego z szyi i korpusów drobiowych.

Material i metody. Materiał do badań stanowiła kiełbasa typu parówkowa, wyprodukowana z mięsa wieprzowego kl. III - był to wariant kontrolny (K), oraz kiełbasy doświadczalne, gdzie 25\% i 50\% mięsa wieprzowego zastąpiono MDOM uzyskanym z szyi (Sz25 i Sz50) i korpusów (W25 i W50) brojlerów kurcząt rasy COB. W kiełbasach oznaczono skład chemiczny oraz barwę L*a*b* w systemie CIE. Wykonano również ocenę sensoryczną metodą 5-punktową. Uzyskane wyniki poddano analizie statystycznej.
} 
Danyluk, B., Bilska, A., Kowalski, R., Danyluk, M. (2016). Evaluation of selected quality characteristics of fine ground sausages of frankfurter type containing MSPM. Nauka Przyr. Technol., 10, 4, \#42. DOI: http://dx.doi.org/ 10.17306/J.NPT.2016.4.42

Wyniki. Na podstawie uzyskanych wyników stwierdzono, że skład podstawowy gotowego wyrobu zmieniał się w zależności od rodzaju i ilości MDOM wprowadzonego do receptury surowcowej. Wprowadzenie MDOM do składu recepturowego badanych wędlin spowodowało istotne obniżenie not takich wyróżników, jak: zapach, barwa przekroju oraz barwa zewnętrzna - ocenianych sensorycznie. $Z$ kolei analiza statystyczna wyników nie wykazała istotnego wpływu częściowej zamiany mięsa wieprzowego mięsem drobiowym oddzielonym mechanicznie na ocenę ogólnej pożądalności modelowych kiełbas.

Wnioski. Częściowa zamiana mięsa wieprzowego mięsem drobiowym oddzielonym mechanicznie uzyskanym z szyi i korpusów brojlerów kurcząt jest możliwa, jednak udział MDOM większy niż $25 \%$ składu recepturowego może niekorzystnie oddziaływać na niektóre wyróżniki jakości sensorycznej. Nie stwierdzono istotnego wpływu zastąpienia mięsa wieprzowego MDOM na fizyczne parametry barwy $a^{*}$ oraz $b^{*}$, niezależnie od rodzaju MDOM oraz ilości substytucji mięsa wieprzowego w recepturze kiełbas parzonych typu parówkowa.

Słowa kluczowe: kiełbasa parówkowa, mięso drobiowe odkostnione mechanicznie (MDOM), jakość

Corresponding address - Adres do korespondencji:

Bożena Danyluk, Instytut Technologii Mięsa, Uniwersytet Przyrodniczy w Poznaniu, ul. Wojska Polskiego 31/33, 60-624 Poznań, Poland, e-mail: danyluk@up.poznan.pl

Accepted for publication - Zaakceptowano do opublikowania:

26.10.2016

For citation - Do cytowania:

Danyluk, B., Bilska, A., Kowalski, R., Danyluk, M. (2016). Evaluation of selected quality characteristics of fine ground sausages of frankfurter type containing MSPM. Nauka Przyr. Technol., 10, 4, \#42. DOI: http://dx.doi.org/10.17306/J.NPT.2016.4.42 